\title{
TREINAMENTO DE FORÇA E SUAS CONTRIBUIÇÕES PARA O CONTROLE DA OBESIDADE EM CRIANÇAS E ADOLESCENTES
}

\section{STRENGTH TRAINING AND ITS CONTRIBUTIONS TO THE CONTROL OF OBESITY IN CHILDREN AND ADOLESCENTS}

\author{
Mailson Ferreira de Souza \\ Bacharel em Educação Física, AGES Paripiranga, Bahia, Brasil \\ E-mail: mailsonz@academico.uniages.edu.br
}

Davi Soares Santos Ribeiro

Mestre em Educação Física, Universidade Federal de Sergipe (UFS)

Docente da AGES Paripiranga, Bahia, Brasil

E-mail: profdavi@live.com

ORCID: https://orcid.org/0000-0001-9816-2566

Recebido: 08/03/2021 - Aceito: 08/03/2021

\begin{abstract}
Resumo
O treinamento de força contribui significativamente na redução da obesidade em crianças e adolescentes. Contudo, ainda é uma prática muito questionada e pouca explorada para esse público, devido à ignorância acerca de sua segurança. $\mathrm{O}$ estudo deste trabalho tem como objetivo discutir os benefícios do treinamento de força na redução da obesidade em crianças e adolescentes. Foi realizada uma pesquisa bibliográfica com abordagem qualitativa. Verificou-se que o treinamento de força possui aspectos relevantes nesse processo, o qual por meio de uma prescrição adequada é possível induzir ao emagrecimento de forma segura, proporcionando benefícios à saúde desse público no contexto biopsicossocial. Conclui-se que o treinamento de força é seguro e eficaz na redução da obesidade em crianças e adolescentes, pois seus benefícios tornam a prática altamente recomendável.
\end{abstract}

Palavras-chave: Obesidade; Prescrição; Treinamento de força.

\begin{abstract}
Strength training contributes significantly to the reduction of obesity in children and adolescents. However, it is still a highly questioned and little explored practice for this public, due to ignorance about its safety. The study of this work aims to discuss the benefits of strength training in reducing obesity in children and adolescents. A bibliographic search was carried out with a qualitative approach. It was found that strength training has relevant aspects in this process, which by means of an appropriate prescription it is possible to safely induce weight loss, providing health benefits to this public in the biopsychosocial context. It is concluded that strength training is safe and
\end{abstract}


effective in reducing obesity in children and adolescents, as its benefits make the practice highly recommended.

Keywords: Obesity; Prescription; Strength training.

\section{Introdução}

A obesidade infantil é um tema pertinente na sociedade que vem causando preocupação no mundo. Segundo Paes, Marins e Andreazzi (2015), a obesidade é um distúrbio metabólico caracterizado pelo acúmulo excessivo de gordura corporal, favorecendo ao desenvolvimento de outras patologias e colocando a saúde da população em risco, principalmente na infância.

Nos últimos anos, tem-se observado um aumento nos índices de sobrepeso e obesidade infantil de forma alarmante. Sob o mesmo ponto de vista, de acordo com a OMS (2016), dados preocupantes são apresentados, sendo que em 2016 o número de crianças de até 5 anos chegou a 41 milhões. De acordo com a mesma agência, estudos indicam que esse número chegará a 75 milhões em 2025.

No Brasil, o contexto da obesidade continua se agravando, onde os números apresentam uma realidade alarmante, sendo que $29,3 \%$ de crianças entre 5 a 9 anos encontram-se em sobrepeso, com cerca de 670,9 mil, sendo 352,8 mil destas com obesidade e 200 mil já em uma situação ainda mais grave, com obesidade mórbida (BRASIL, 2019).

Nesse contexto, o exercício físico torna-se uma ferramenta eficaz no combate a obesidade infantil. De acordo com o Colégio Americano de Medicina do Esporte (ACSM, 2009), o exercício físico aumenta o gasto calórico e reduz o ritmo de perda de massa magra. Nessa vertente, destaca-se o treinamento de força contra a obesidade infantil, visando o controle de peso, composição corporal, e ainda auxiliando no combate e na prevenção das síndromes metabólicas.

O Treinamento de Força é uma ampla gama de modalidades de treinamentos, dos quais abrangem exercícios corporais com pesos, uso de tiras elásticas, pliometria e corrida em ladeiras (FLECK; KRAEMER, 2017).

Nesse contexto, o estudo tem como objetivo discutir os benefícios do treinamento de força para o controle da obesidade em crianças e adolescentes. 


\section{Revisão Bibliográfica}

\section{Treinamento de Força}

O Treinamento de Força é definido como uma prática de exercícios físicos em que o indivíduo precisa superar determinada carga de resistência que the é imposta através da contração voluntária da musculatura esquelética, podendo utilizar pesos livres, máquinas, fitas elásticas e até o próprio peso corporal (FLECK; KRAEMER, 2001).

Ele tem sendo indicado para tratamento de pessoas com sobrepeso, e principalmente, com obesidade, por ser uma prática eficaz na redução da gordura corporal (Ferreira et al., 2006).

Quando se trata de crianças e adolescentes ainda há receio sobre o treinamento de força. Braga et al. (2008) salienta que crianças e adolescentes não praticam a modalidade, pelo fato de existirem leigos e até profissionais da saúde que consideram ser uma prática inadequada para o crescimento e desenvolvimento de crianças e adolescentes, pois associam o Treinamento de Força ao fisiculturismo e ao levantamento de peso olímpico.

Fleck e Kraemer (2017) refutam essa ideia, afirmando que há estudos que comprovam que o treinamento de força é benéfico para crianças e adolescentes, no qual há uma contribuição na aceleração do crescimento longitudinal, espessura óssea, além de liberar muitos hormônios, assim como a testosterona e GH (hormônio do crescimento).

Nesse contexto, a Academia Americana de Pediatria (AAP, 2008) ressalta que o treinamento de força, além de não oferecer riscos à saúde e integridade física de crianças e adolescentes, proporciona benefícios à saúde dessa população, reforçando a importância da supervisão de um profissional da área.

O treinamento de força possui várias finalidades, podendo ser utilizado para manutenção e aumento do metabolismo, sendo que estes interferem diretamente na manutenção ou aumento de massa muscular, e na redução da gordura corporal. Vale lembrar que também é possível realizar treinamento com peso livre, isso abre o leque para diversas práticas de exercício físico sem a utilização de equipamentos (SILVA, 2004). 
Revista Multidisciplinar do Nordeste Mineiro, v.1,

2021/01

ISSN 2178-6925

Portanto, verifica-se que o treinamento de força oferece condições para que crianças e adolescentes possam treinar, a partir de uma manipulação correta e consciente. Logo, estes poderão adquirir melhores condições de saúde e qualidade de vida, através de um melhor condicionamento físico, reduzindo os riscos de doenças cardiovasculares e melhorando sua disposição e coordenação motora, além de outros benefícios (RUAS et al., 2014).

\section{Prescrição do Treinamento de Força para Crianças e Adolescentes}

A prescrição do treinamento deve descrever o treino através de componentes essenciais, assim como os tipos de exercícios, quantidade de séries e repetições, intervalos, cadência, entre outras variáveis que se adequem ao caso (Lima e Chagas, 2008).

O emagrecimento induzido pelo treinamento de força proporciona melhoria na postura e aumento de massa muscular, e para isso, se faz necessário um planejamento consciente e um acompanhamento profissional, com o intuito de controlar a empolgação desse público infantil para evitar possíveis exageros durante a prática que possam comprometer sua integridade física (FLECK, 2001).

Para Weineck (1999), é importante o profissional dominar os princípios do treinamento esportivo, pois dessa forma é possível melhor direcionar as atividades a serem feitas, prezando pela segurança, e possibilitando atingir resultados mais significativos. Além disso, o treinamento tende a ser mais organizado, podendo dividi-lo em etapas, cada uma com objetivo de aprimorar determinadas capacidades físicas ou de melhorar ações técnicas táticas, em casos de esportes coletivos e individuais.

Destarte, Fleck e Kraemer (2017), aconselha que o treinamento de força voltado para o público infantil deve utilizar uma abordagem que favoreça a realização de todas as atividades, tornando o ambiente mais divertido, mas sempre priorizando a segurança. Assim, o profissional consegue envolver a criança no treinamento, evitando uma prática monótona, fato que poderia causar desânimo na criança ou até desistência.

Ao pensar no treinamento de força voltado para crianças e adolescentes, elucida a importância da idade no processo da prescrição, sendo fundamental para 
que o praticante tenha um bom desempenho e com segurança. Nesse sentido, esses indivíduos precisam ter um bom nível de desenvolvimento corporal, onde a postura, equilíbrio, coordenação motora e autocontrole são capacidades físicas essenciais para treinar de forma segura e eficiente (MCCAMBRIDGE; STRICKER, 2008).

De acordo com McCambridge e Stricker (2008), não é seguro a prática do treinamento de força com crianças com menos de 7 anos de idade, pois estes começam a desenvolver sua coordenação motora a níveis maduros entre 7 a 8 anos de idade. Os autores ressaltam que é necessário a criança possuir outras habilidades, assim como bons níveis de concentração, entender as orientações passadas pelo profissional. Desse modo, a realização dessa prática só será eficaz se a criança possuir essas capacidades.

Para Roberts e Weider (1994), dependendo da idade da criança e de sua maturação psicológica, o treinamento para esse público pode ter um tempo de duração entre 20 a 60 minutos por sessão, na qual as séries podem ser até 3, com intensidade baixa, suficiente para a realização de 6 repetições, com frequência de 3 vezes por semana. Entretanto, é necessário frisar que esses números não devem ser fixos, cabendo ao profissional analisar a realidade do indivíduo para escolher as variáveis mais adequadas para o caso.

A escolha dos exercícios é uma etapa importante, o qual deve observar fatores como: objetivo, necessidade e característica do indivíduo. Com isso, vale destacar que os exercícios multiarticulares são considerados mais eficientes para trabalhar com esse público, pois há um recrutamento de um maior número de musculaturas, envolvendo mais de uma articulação, sendo eficiente para um melhor desenvolvimento motor (ROBERTS; WEIDER, 1994).

Tendo isso em vista, podem ser efetuados exercícios que trabalhem a coordenação motora, equilíbrio e atenção, possibilitando uma condição mais favorável ao treinamento de força voltado para o emagrecimento dessa população com obesidade, da qual requer um melhor condicionamento físico para ter eficácia nesse processo (SANTARÉM, 2014).

Ao elaborar uma prescrição para crianças e adolescentes iniciantes, o profissional deve priorizar exercícios mais simples e ir acrescentando os mais 
complexos, conforme for progredindo no treinamento e adquirindo mais confiança e consciência corporal. Após a familiarização com a prática, poderia ser incluído exercícios multiarticulares, em uma periodização que contenha os principais músculos por sessão, e posteriormente, podendo utilizar exercícios monoarticulares, a depender da estratégia (UGHINI et al., 2011).

Bompa (2002), sugere que para estruturar uma boa prescrição para esse público pode ser incrementado no programa de treinamento exercícios com pesos livres combinados com outros meios de treinamento, como por exemplo, utilizar medicina ball, aparelhos e saltos direcionados. Dessa forma poderia agregar atividades com variáveis diferentes, tornando o treinamento mais ativo, sendo ideal para esse público.

Santarém (2014) enfatiza que os aparelhos e máquinas das academias são desenvolvidos para o público adulto. Diante disso, não é recomendado prescrever exercícios para crianças de baixa estatura nessas estruturas, pois a biomecânica não é adequada e pode comprometer o bom desempenho e até a integridade física.

É válido destacar que os exercícios pliométricos proporcionam ganhos consideráveis de força em crianças e adolescentes, os quais fazem parte do cotidiano, sendo realizados saltos através de brincadeiras. Sendo assim, o profissional pode incluir esses exercícios no programa de treinamento para proporcionar uma vivência mais dinâmica do treinamento de força (UGHINI et al., 2011).

No que se refere ao treinamento de força, Santarém (2014) reforça que existem restrições para adolescentes com cardiomiopatias graves, hipertensão pulmonar, síndrome de Marfan. Também em indivíduos submetidos a sessão de quimioterapia, pois há um risco cardiovascular por conta dos efeitos cardiotóxicos, sendo assim, deve haver maior cautela, e em casos de epilepsia é necessário um acompanhamento, assim como em casos de sobrepeso, onde o paciente apresenta baixar níveis de força e condição física.

Diante disse aspecto discutido, as características da prescrição para crianças e adolescentes na fase inicial do treinamento de força, sendo a adaptação uma fase essencial nesse processo. Faigenbaum (2003) explica que as séries com 
realização de 8 a 12 repetições, com cargas leves, com frequência de 2 a 3 vezes por semana, sempre com um dia de repouso após o dia de treino. Conforme eles vão se adaptando, é recomendado atualizar o treinamento com uma nova prescrição, da qual deve conter ajustes nas variáveis, de modo que proporcione novos estímulos.

Faigenbaum (2003), recomenda que após a sessão de treinamento, eles possam dar uma volta, caminhando lentamente a fim de relaxar o corpo, e normalizar sua temperatura corporal e frequência cardíaca, além de realizar alongamentos. O autor ainda destaca a importância desse momento entre o praticante e o treinador, no qual é necessário haver um feedback entre ambos, para compreender melhor como o praticante se sente, e procurar identificar possíveis fragilidades nesse processo.

Contudo, é necessário observar algumas recomendações que a literatura dispõe para prescrever um treinamento para esse público, na qual os métodos de treinamento podem variar conforme a faixa etária dos indivíduos. Desse modo, Pinto (2014), relata que a intensidade seria modificada ao longo da periodização do treinamento, a fim de melhorar o rendimento do praticante em longo prazo. E diante disso, é possível promover estímulos necessários para que a criança ou o adolescente possa perder mais peso.

Sendo assim, no período entre 8 a 10 anos, deve ser prescrito um treinamento mais dinâmico, pelo fato da criança não possuir alta capacidade anaeróbica, com pouca solicitação de força, poucas repetições, uma série por exercício e sempre descansando bem. É importante recomendar poucas sessões em períodos iniciais, podendo prescrever até 1 sessão por semana, embora 2 sessões em dias alternados proporcionam melhores resultados na adaptação (PINTO, 2014).

Posteriormente, na fase entre 10 a 13 anos, onde ocorre um desenvolvimento estrutural desses indivíduos, é indicado prescrever exercícios que melhorem sua funcionalidade e seus níveis de força, assim como exercícios gerais, que envolvam puxar e empurrar. E no processo de perda de peso é interessante praticar outras modalidades a fim de aumentar o gasto calórico, podendo ser sugerido a natação, entre outras modalidades (SANTARÉM, 2014). 
Em seguida, na prescrição para praticantes entre 13 a 16 anos pode optar por exercícios multiarticulares, sempre enfatizando um descanso adequado, pois há uma maior necessidade quando comparado aos adultos, com as sessões variando entre 20 a 50 minutos, até 3 vezes por semana e somente em dias alternados. A necessidade de fortalecer a lombar e abdômen, na qual elas possuem papel importante para manter o equilíbrio corporal e uma boa postura (BENEDET, 2013).

De acordo com Bompa (2002), a fundamentação específica para os ganhos de força deve ser inserida na prescrição entre 11 a 14 anos. Dessa forma, pretende-se que o praticante desenvolva níveis significativos de força, sugerindo um treinamento que fortaleça e desenvolva os membros superiores, a região do tronco, bem como membros inferiores, abdômen e lombar.

Por outro lado, o profissional deve ter muita atenção no comportamento do praticante, sendo necessário sempre estar dialogando com ele, de forma a motiválo dentro desse processo no combate a obesidade, compreendida como uma condição complexa. Nesse sentido, a motivação é considerada um elemento fundamental, do qual pode influenciar nos resultados dos praticantes do treinamento de força (OLIVEIRA; GALLAGHER; SILVA, 1995).

Do mesmo modo, Fleck e Kraemer (2017), trazem suas contribuições nesse viés, reforçando através de orientações com base científica, para a prescrição do treinamento de força em diferentes faixas etárias. Para idades entre 5 a 7 anos, a criança deve iniciar com exercícios básicos, com pouco uso de cargas, ou usando o próprio peso corporal, dando ênfase no desenvolvimento das técnicas de execução. Os autores ainda recomendam a progressão dos exercícios calistênicos para uso de cargas leves, dentro de um programa de treinamento de baixo volume.

Com base nisso, nas orientações para crianças entre idades de 8 a 10 anos, sugere-se o aumento do volume e carga de treino de forma gradativa, sempre visando aprimorar a técnica. Nessa fase é importante perceber o nível de estresse do exercício, dos quais ainda devem ser de pouca complexidade (FLECK; KRAEMER, 2017).

Em seguida, se tratando da faixa-etária de 11 a 13 anos deve continuar progredindo a intensidade nos exercícios, enfatizando a técnica de execução, a fim 
de obter melhores resultados e minimizar o risco de lesões que podem ser causadas por execução dos exercícios de forma inadequada. Diante disso, após uma progressão significativa nas técnicas de execuções, deve inserir exercícios mais avançados. Torna-se importante destacar a utilização de poucas cargas nas fases de transição entre os exercícios (FLECK; KRAEMER, 2017).

Com base nesses estudos de Fleck e Kraemer (2017), verifica-se a necessidade de uma progressão no nível de treinamento para adolescentes entre 14 a 15 anos, pode ser incluído métodos mais específicos, desde que esteja de acordo com o princípio de treinamento esportivo. A técnica é um componente fundamental em todas as fases, principalmente nesse avanço do nível de treinamento, o qual recomenda que o volume seja aumentado.

Aos 16 anos em diante é a fase em que todo o processo do indivíduo passa a servir como base para o início de programas de treinamento de força para adultos, na qual os níveis de volume e intensidade podem ser bem elevados, além de métodos que podem ser utilizados com objetivos específicos. Sendo assim, independente do objetivo do praticante, é necessário que a criança ou adolescente praticante do treinamento de força, tenha desempenho significativo durante cada fase, possibilitando transições entre elas de forma segura e contínua obtendo bons resultados (FLECK; KRAEMER, 2017).

Dentro dessas recomendações, Fleck e Kraemer (2001), destacam algumas mais direcionadas para crianças e adolescentes com obesidade, no qual o descanso no treino aeróbico deve ser ativo, e no treino anaeróbico é sempre importante grandes períodos de descanso. Do mesmo modo, a duração do treino anaeróbio deve ser reduzida, enquanto o aeróbico pode ter maior volume.

Nesse processo é importante ocorrer uma variação ou incremento nos exercícios, podendo ser realizada entre 2 a 4 semanas de treinamento. É necessário elucidar que devem ser mantidos os exercícios principais para cada grupo muscular, com objetivo de propiciar uma melhor adaptação desses movimentos. Além disso, é válido variar exercícios que envolvam o mesmo grupo muscular, aumentando a carga e variando a quantidade de séries e repetições, podendo utilizar de 1 a 3 séries, entre 6 a 20 repetições máximas, além da 
possibilidade de variar os dias de treino com intensidades alta e baixa (FLECK; KRAEMER, 2001).

Considerando o avanço gradativo da sobrecarga no treinamento de força para esses indivíduos, é imprescindível que o treinador tenha devido controle para a realização dos exercícios nesse processo do qual ocorre o aumento da intensidade. De acordo com Faigenbaum (2003), o teste de repetição máxima (RM), pode ser aplicado em crianças pré-púberes, de ambos os sexos com segurança, com a intenção de mensurar sua força muscular, desde que o praticante já esteja familiarizado com a prática.

É fundamental enfatizar o fator genético como um dos principais fatores que modulam os efeitos provenientes do treinamento de força para todos os indivíduos de acordo com suas características, do qual é responsável por influenciar diretamente no desempenho da prática (MCARDLE; KATCH; KATCH, 1998). Dessa forma, os estímulos e resultados tendem a variar de acordo com cada indivíduo, ainda que o mesmo treinamento tenha sido feito por diferentes indivíduos.

Santarém (2014) reforça que existem restrições para a prática do treinamento de força, assim como adolescentes que possuam cardiomiopatias graves, hipertensão pulmonar síndrome de Marfan. Em indivíduos fazendo quimioterapia há um risco cardiovascular por conta dos efeitos cardiotóxicos, sendo assim, deve haver maior cautela, e em casos de epilepsia é necessário um acompanhamento, assim como em casos de sobrepeso, onde o paciente apresenta baixar níveis de força e condição física.

Conforme Guedes e Guedes (1995), o treinamento voltado para melhorar o desempenho motor, bem como induzir a redução da gordura corporal de crianças e adolescentes, pode proporcioná-los uma condição física mais favorável à saúde, fato que influenciará diretamente em suas experiências de vida. Além de contribuir com uma melhor qualidade de vida.

Para isso, é necessário que esses indivíduos continuem com um estilo de vida mais ativo e saudável, no qual requer uma boa qualidade de sono, evitar o consumo de álcool ou drogas ilícitas, e sempre priorizando uma alimentação 
Revista Multidisciplinar do Nordeste Mineiro, v.1,

adequada, aliado a prática de exercícios, assim como o próprio treinamento de força (OLIVEIRA, 1996).

\section{Considerações Finais}

O treinamento de força contribui no desenvolvimento motor e psicológico em crianças e adolescentes, sendo eficaz na redução do excesso de gordura. Para isso, faz-se necessário a presença de um profissional de Educação Física para a prescrição e orientação segura, preservando a saúde e integridade física desse público.

Ele contribui significativamente no emagrecimento, em benefícios à saúde cardiovascular, no desenvolvimento estrutural, bem como aumento da massa muscular, fortalecimento das estruturas ósseas e articulares, assim reduzindo os riscos de lesionar.

\section{Referências}

AAP. American Academy of Pediatrics Council on Sports Medicine and Fitness, McCambridge TM, Stricker PR. Strength training by children and adolescents.

Pediatrics 2008;121:835-40.

ACSM, American College of Sports Medicine. American College of Sports Medicine position stand. Progression models in resistance training for healthy adults.

Medicine and science in sports and exercise, v. 41, n. 3, p. 687, 2009.

BENEDET, Jucemar. Treinamento resistido para crianças e adolescentes.

Arquivos Brasileiros de Ciências da Saúde, 2013.

BOMPA, Tudor O. Periodização: Teoria e Metodologia do Treinamento. 4. ed. São Paulo: Phorte, 2002. 
Revista Multidisciplinar do Nordeste Mineiro, v.1,

2021/01

ISSN 2178-6925

BRAGA, F. et al. Programas de Treinamento de Força para Escolares sem uso de Equipamentos. Revista Eletrônica da Ulbra São Jerônimo, v.3. 2008.

BRASIL. Ministério da Saúde. Secretaria de Políticas de Saúde. Atlas da obesidade infantil no Brasil. Brasília, DF, 2019.

FAIGENBAUM, A. Youth resistance training. President's Council on Physical fitness and Sports. 2003.

FERREIRA, S. et al. Aspectos etiológicos e o papel do exercício físico na prevenção e controle da obesidade. Revista de Educação Física, Viçosa, 2006; n.133, p. 15-24.

FLECK, S. J.; KRAEMER, W. J. Fundamentos do treinamento de força muscular. 4ํe ed. Porto Alegre: Artmed, 2017.

FLECK, S. J; KRAEMER, W.J. Fundamentos do treinamento de força para jovens atletas. São Paulo: Manole, 2001.

GUEDES, D. P.; GUEDES, J. E. R. P. Aptidão física relacionada à saúde de crianças e adolescentes: avaliação referenciada por critério. Revista Brasileira de Atividade Física e Saúde, Londrina, v.1, n.2, p.27-38, 1995.

LIMA, F.V.; CHAGAS, M.H.; Musculação: variáveis estruturais. Belo Horizonte: Casa da Educação Física, 2008.

MARTINS, G.A. \& PINTO, R.L. Manual para elaboração de trabalhos acadêmicos. São Paulo: Atlas, 2001.

MCARDLE, W. D.; KATCH, F. I.; KATCH, V. L. Fisiologia do Exercício: energia, nutrição e desempenho humano. 4.ed. Rio de Janeiro: Guanabara Koogan, 1998. 
Revista Multidisciplinar do Nordeste Mineiro, v.1,

MCCAMBRIDGE TM, STRICKER PR. Strength training by children and adolescents. American Academy of Pediatrics Council on Sports Medicine and Fitness. Pediatrics 2008;121(4):835-40.

OLIVEIRA, A. R. de; GALLAGHER, J. D.; SILVA, S. G. Musculação em crianças: uma meta análise. Revista da Associação dos Professores de Educação Física de Londrina, Londrina, v.10, n.18, p.70-76, 1995.

OLIVEIRA, A. R. Fatores influenciadores na determinação do nível de aptidão física em crianças. Synopsis, Curitiba, v.7, p.48-62, 1996.

OMS. Organização Mundial da Saúde. Ending childhood obesity: report of the commission on. World Health Organization, 2016.

PAES, Santiago; MARINS, João; ANDREAZZI. Efeitos metabólicos do exercício físico na obesidade infantil: uma visão atual. Revista Paulista de Pediatria. 2015;33(1):122-129.

PINTO, Ronei S. et.al. Treinamento de força para crianças e adolescentes: adaptações, riscos e linhas de orientação. Brazilian Journal of Motor Behavior, vol.8, N 1, 2014.

ROBERTS, S.; WEIDER, B. Strength and Weight Training for Young Athletes. Chicago: Contemporary Books, 1994.

RUAS, C. et al. Treino de força para crianças e adolescentes: adaptações, riscos e linhas de orientação. Brazilian Journal of Motor Behavior, v. 8, n. 1, 2014.

SANTARÉM, José Maria. Orientações para o treinamento resistido com base em evidências. Med. Sport 15 (3): 147 - 162, 2014.

SILVA. F. M. Treinamento de força na infância e na juventude. Campinas, 2004. 
Revista Multidisciplinar do Nordeste Mineiro, v.1, 2021/01

ISSN 2178-6925

SILVA, E. L. da; MENEZES, E. M. Metodologia da pesquisa e elaboração de dissertação. 4. ed. Florianópolis: UFSC, 2005.

UGHINI, C. et al. Treinamento de força em crianças: segurança, benefícios e recomendações. CONEXÕES: Revista da Faculdade de Educação Física da UNICAMP, v. 9, n. 2, 2011.

WEINECK, J. Treinamento Ideal. São Paulo: Manole,1999. 
Revista Multidisciplinar do Nordeste Mineiro, v.1, 2021/01

ISSN 2178-6925 
Revista Multidisciplinar do Nordeste Mineiro, v.1, 2021/01

ISSN 2178-6925 
Revista Multidisciplinar do Nordeste Mineiro, v.1, 2021/01

ISSN 2178-6925 